\title{
Effect of the Vacuum Vessel on the Measurements of Mirnov Oscillation in TCABR
}

\author{
M. Y. Kucinski and Yu. K. Kuznetsov \\ Instituto de Física da Universidade de São Paulo \\ C. P. 66318, CEP 05315-970, São Paulo, SP, Brazil
}

Received on 26 June, 2001

\begin{abstract}
Magnetic field due to instabilities in plasma is expected to be reflected in the conductive vessel wall. We evaluate the reflection effect in the Brazilian tokamak TCABR. The magnetic field due to the plasma is represented by a toroidal harmonic function in vacuum and the reflected field is written as a series of harmonic functions in vacuum, generated in the external region. The conclusion is that a signal originated in the plasma may be strongly deformed, close to the wall. Even far from the wall, around the plasma, the effect of the wall may still be considerable.
\end{abstract}

\section{Introduction}

The magnetic field due to Mirnov oscillations in a tokamak plasma is reflected in the conductive vessel wall. Therefore, the measurement in the probes is a superposition of the field produced by the plasma and its reflection in the wall. If Mirnov probes are assembled near the wall we have the problem of discerning the instability field originated in the plasma.

In present work we calculate the reflected field in TCABR tokamak [1].

For simplicity, the magnetic field due to plasma is represented here by an axisymmetric toroidal harmonic function in vacuum and the reflected field is written as a series of harmonic functions in vacuum, generated in the external region. In fact, the Mirnov oscillations are helical. But, the effect of vacuum vessel can not be very different for a helical field as compared with an axisymmetric one. The coefficients of the series are determined, assuming that the wall is a magnetic surface, or, equivalently, that the magnetic field has no component normal to the wall. TCABR chamber has rectangular cross section. A method is developed in order to solve a boundary value problem when the boundary is not a circular torus. Presently, a set of Mirnov coils are fixed near $(\approx 1.9 \mathrm{~cm})$ the wall. There is a project for another set of coils to be placed around the plasma. We estimate the field in the positions of these two sets.

The conclusion is that a signal originated in the plasma may be strongly deformed, close to the wall.

In section II, boundary conditions are formally written in terms of harmonic functions, by using conventional toroidal coordinates. In section III, idea of best approximation in the mean is introduced in order to establish boundary conditions over surfaces with any shape. The method is applied to find field reflection in a tokamak vessel, in section IV, and specifically in TCABR, in section V. Conclusions are drawn in section VI.

\section{Boundary Conditions}

The magnetic field in the vacuum is written

$$
\vec{B}=\overrightarrow{B^{P}}-\overrightarrow{B^{R}}
$$

where the suffix $P$ denotes field associated to instability mode in the plasma and $R$, field reflected in the wall. If the field is axially symmetric, it can be written in terms of flux function $\Psi$ as

$$
\vec{B}=\nabla \Psi \times \frac{\overrightarrow{e_{\varphi}}}{r} ; \quad \Psi=\Psi^{P}-\Psi^{R}
$$

where $(r, \varphi, z)$ are the usual cylindrical coordinates and $\overrightarrow{e_{\varphi}}$ is the unity vector in the toroidal direction.

Toroidal coordinates $(\eta, \theta, \varphi)$ are defined by the following relationships with the cylindrical coordinates:

$$
r=\frac{R_{0} \sinh \eta}{\cosh \eta-\cos \theta} \quad \text { and } \quad z=\frac{R_{0} \sin \theta}{\cosh \eta-\cos \theta},
$$

where $R_{0}$ is he radius of the circular axis and $\varphi$ is the toroidal angle.

In this paper we assume axial symmetry because the calculation becomes considerably easier and we do not expect significant difference in order of magnitude of reflection if there is no symmetry.

Also, we assume up-down symmetry.

The plasma field is taken as a single toroidal harmonic:

$$
\Psi^{P}=\Psi_{m_{0}}^{i}
$$


where

$$
\Psi_{m_{0}}^{i}=\frac{\sinh \eta}{\sqrt{\cosh \eta-\cos \theta}} P_{m_{0}-1 / 2}^{1}(\cosh \eta) \sin \left(m_{0} \theta\right) .
$$

We use $P_{m}^{n}$ and $Q_{m}^{n}$ for associated Legendre functions of first and second kinds, respectively.

The reflected field is considered as a superposition of finite number $(\mathrm{N})$ of regular toroidal harmonics

$$
\Psi^{R} \approx \sum_{m=1}^{N} \gamma_{m} \Psi_{m}^{e}
$$

where

$$
\Psi_{m}^{e}=\frac{\sinh \eta}{\sqrt{\cosh \eta-\cos \theta}} Q_{m-1 / 2}^{1}(\cosh \eta) \sin \left(m_{0} \theta\right) .
$$

The border is described by a relation in the form

$$
\cosh \eta=x_{s}(\theta)
$$

This is a convenient form as $x_{s}$ is a single-valued function of $\theta$.

Instead of (5), we can write the reflected field as

$$
\begin{gathered}
\Psi^{R} \approx \sum_{k} c_{k} F_{k}(\cosh \eta, \theta) ; \quad F_{k}=\sum_{\ell} \beta_{k, \ell} \Psi_{\ell}^{e} ; \\
\beta_{k, \ell}=\text { constant. }
\end{gathered}
$$

$F_{k}$ is a linear combination of $\Psi_{\ell}$. Therefore, equation (II) is a different assembly of the same functions.

We assume that on the wall the normal component of the magnetic field is zero; this is equivalent to state that the border is a magnetic surface (that $\Psi$ is constant on the wall).

We use small letters to represent functions of $\theta$ on the border:

$$
\Psi=\psi(\theta) ; \quad F_{k}=f_{k}(\theta) .
$$

Our boundary value problem becomes finding $c_{k}$ and $\beta_{k, \ell}$ in order to have the best approximation

$$
\psi^{P} \sim \psi^{R} \approx \sum_{k} c_{k} f_{k}(\theta)
$$

or

$$
b_{n}^{P} \sim b_{n}^{R} \approx \sum_{k} c_{k} b_{n k}(\theta)
$$

The prefix $n$ denotes normal component.

There is no ready made theory for boundary value problems when the boundary is not coincident with conventional coordinate surfaces. In TCABR, the vessel cross section is rectangular. In order to solve the problem, idea of best approximation in the mean is taken from [2] and developped in the next section.

\section{Best approximation in the mean}

We define distance $\sigma$ between two functions $f(\theta)$ and $g(\theta)$, defined on the border, by

$$
\sigma^{2} \equiv \int_{0}^{2 \pi}|f(\theta)-g(\theta)|^{2} \rho(\theta) d \theta ; \quad \rho(\theta)>0
$$

where $\rho$ is a positive weight function.

Then, the distance between the functions $\psi^{P}(\theta)$ and $\sum_{k=1}^{N} c_{k} f_{k}(\theta)$ is given by

$$
\sigma^{2}=\int_{0}^{2 \pi}\left|\psi^{P}-\sum_{k=1}^{N} c_{k} f_{k}(\theta)\right|^{2} \rho(\theta) d \theta
$$

$\sigma$ is a measure of the error caused by representing the reflected field by a sum of finite number $\mathrm{N}$ of harmonics. The approximation is considered as the best in the mean, for a fixed $\mathrm{N}$, when the values of $c_{k}$ are chosen to minimize the distance $\sigma$. These can be obtained by evaluating

$$
\frac{\partial \sigma^{2}}{\partial c_{\ell}}=0 \quad \text { for } \quad \ell=1,2, \cdots, N
$$

We get

$$
\int_{0}^{2 \pi} \psi^{P}(\theta) f_{\ell}(\theta) \rho(\theta) d \theta-\sum_{k=1}^{N} c_{k} \int_{0}^{2 \pi} f_{k}(\theta) f_{\ell}(\theta) \rho(\theta) d \theta=0
$$

We define scalar product of two functions $f$ and $g$ on the surface as

$$
(f, g) \equiv \int_{0}^{2 \pi} f(\theta) g(\theta) \rho(\theta) d \theta
$$

and write (13) as

$$
\left(\psi^{P}, f_{\ell}\right)-\sum_{k=1}^{N} c_{k}\left(f_{k}, f_{\ell}\right)=0
$$


If the $f_{k}$ 's are orthonormalized in the sense

$$
\left(f_{k}, f_{\ell}\right)=\delta_{k, \ell},
$$

where $\delta_{k, \ell}$ is the Kronecker symbol, $c_{\ell}$ is obtained straightforwardly as

$$
c_{\ell}=\left(\psi^{P}, f_{\ell}\right)
$$

If $f_{k}$ is simply taken as the harmonic function (6), on the surface, i. e., $\beta_{k, \ell}=\delta_{k, \ell}$, it is necessary to invert the matrix $\left(f_{k}, f_{\ell}\right)$ in order to find the coefficients.

Advantages of using orthonormalized functions are that (i) there is no problem of matrix inversion; (ii) Gram-Schmidt method can be used to construct, one by one, an orthonormalized set of functions; and (iii) it is not necessary to fix, a priori, the number of terms $N$. Each time a new term is added, the fit is checked, either visually, drawing a graph or by evaluating $\sigma$. If the result is not satisfactory we proceed with new term.

A disadvantage of using orthogonal functions is that the Gram-Schmidt orthogonalization may introduce numerical errors if $\mathrm{N}$ is too large.

Disadvantages of using non-orthogonal functions are that (i) the matrix to be inverted may be illconditioned; and (ii) $N$ must be fixed a priori. If the approximation is not satisfactory it is not a question of just adding a new term. The calculation has to be performed all over again.

\section{Field reflection in tokamak wall}

We use the Gram-Schmidt method to construct, one by one, an orthonormalized set of functions $f_{k}$, in the form (II):

$$
f_{k}=\sum_{\ell=1}^{k} \beta_{k, \ell} \psi_{\ell}^{e} ; \quad k=1,2, \cdots, N
$$

with the condition of orthonormality (16). $\psi_{1}^{e}$ is normalized to be $f_{1}$ and each one of the other functions $f_{k}$ are built to be orthogonal to the previous ones.

In this work, we used weight function $\rho(\theta)=1$.

Once $c_{\ell}$ is obtained by using equation (17), we may write $\Psi^{R}$ in terms of the original harmonics as in equation (5).

We use equation (II) and get:

$$
\sum_{m=1}^{N} \gamma_{m} \psi_{m}^{e}=\sum_{k=1}^{N} c_{k} f_{k}=\sum_{k=1}^{N} c_{k} \sum_{\ell=1}^{k} \beta_{k, \ell} \psi_{\ell}^{e}=\sum_{\ell=1}^{N} \psi_{\ell}^{e} \sum_{k=\ell}^{N} c_{k} \beta_{k, \ell}
$$

and, therefore,

$$
\gamma_{\ell}=\sum_{k=\ell}^{N} c_{k} \beta_{k, \ell}
$$

\section{Application to TCABR toka- mak}

The vacuum vessel of TCABR has rectangular cross section. The dimensions are: $R_{a}=0.845 \mathrm{~m}$ (external radius), $R_{b}=0.4 \mathrm{~m}$ (internal radius) and $H=0.52 \mathrm{~m}$ (height).

The plasma minor radius is $a=0.18 \mathrm{~m}$ and the major radius is $R_{p}=0.615 \mathrm{~m}$.

Magnetic sensors (22 two-component magnetic probes) are assembled in a rectangular surface, at 1.9 $\mathrm{cm}$ from the wall, as shown in Fig. 1.

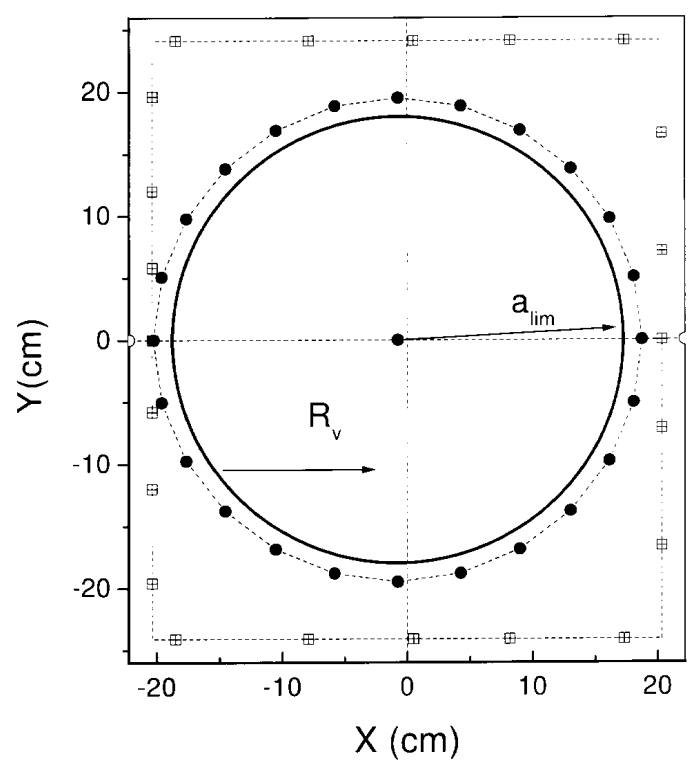

Figure 1. Mirnov probe location in TCABR. The probes are represented by $\square$ 's (present set) and the designed set (in project) is shown by •'s. The outer rectangle represents the vessel and the circle, the plasma surface.

New sensor set that is planned for TCABR includes 24 magnetic probes, equally spaced, located at 
a circular surface around the plasma, with minor axis $\rho_{p}=0.2015 \mathrm{~m}$ and major axis $R_{p}=0.615 \mathrm{~m}$.

Results of calculations for $m_{0}=2$ are shown in Figs. 2 to 7 .

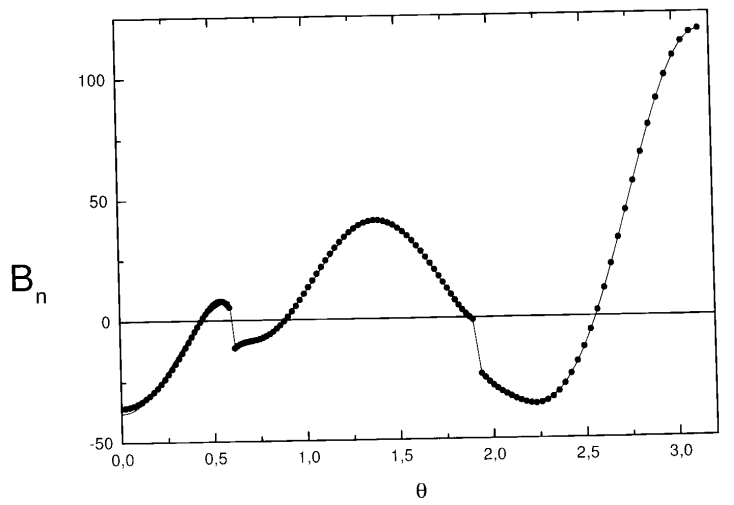

Figure 2. Comparison between the normal component of $\vec{B}$, on the boundary, due to the plasma (solid line), and the field reflected in the vacuum vessel (dots). 16 toroidal harmonics are used to calculate the reflected field in reply to single $m_{0}=2$ harmonic generated by the plasma.

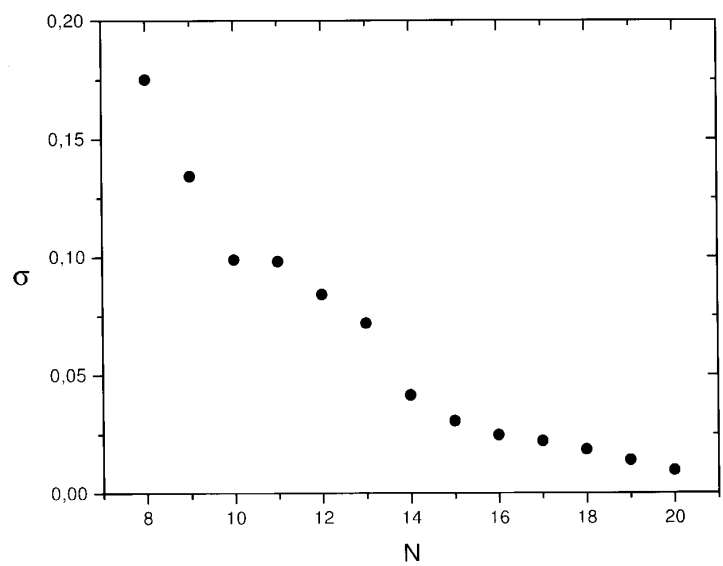

Figure 3. Mean error $\sigma$ in the reflected field determination, on the boundary, as a function of the number $\mathrm{N}$ of harmonics.

Figs 2 and 3 show the convergency of the boundary value method described here. In Fig. $2, b_{n}^{P}$ and $b_{n}^{R}$, on the boundary, are plotted as function of polar angle $\theta$. As $b_{n}$ is not continuous function of $\theta$, the convergency is not uniform. Even so, for $N \gtrsim 16$, normal components of plasma, and reflected fields are nearly coincident. Fig. 3 shows the method accuracy $\sigma$ as a function of the number $\mathrm{N}$ of harmonics. $N \gtrsim 14$ is enough to obtain satisfactory result.

By using non orthogonal set of harmonic functions we came to face problem of inversion of ill-conditioned matrix for $N \geq 16$.

According to equation (5), the reflected magnetic field in the position of each probe $i$ is

$$
\vec{B}^{R}\left(\vec{r}_{i}\right) \sim \sum_{k=1}^{N} \gamma_{k} \vec{B}_{k}\left(\vec{r}_{i}\right)
$$

In order to estimate the importance of each harmonic in the reflected field, we define an average amplitude of mode $k$ (harmonic $k$ ) as

$$
A(k) \equiv\left[\sum_{i=1}^{N_{p}} \gamma_{k}^{2}\left|\vec{B}_{k}\left(\vec{r}_{i}\right)\right|^{2}\right]^{1 / 2} \frac{1}{N_{p}}
$$

where $N_{p}$ is the number of probes.

Figs. 4 and 5 refer to measurements in the probes placed on a square surface, near the wall (present set of sensors) and Figs. 6 and 7 to measurements in the probes equally spaced on a circular surface, farther from the wall (in project).

$A(k)$ is very scattered in Fig. 4, what means that, in reply to a single mode produced by a plasma, the reflected field is generated with a complicated structure. Thus, interpretation of measurements requires reconstruction of the vacuum field, taking into account the effect of the vacuum vessel.

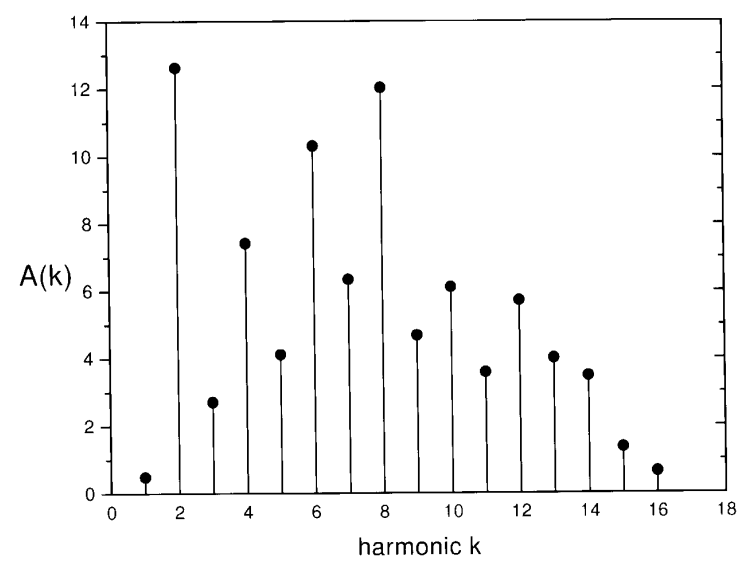

Figure 4. Spectrum of harmonics in reflected magnetic field at the position of the present set of probes.

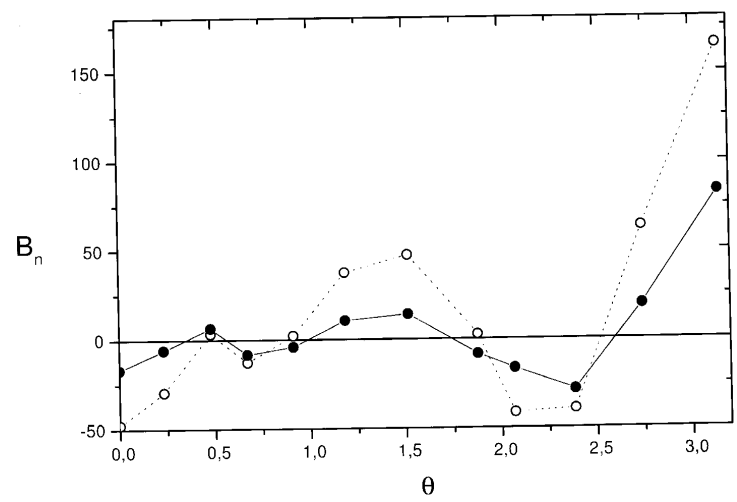

Figure 5. Magnetic field normal to the wall, in the position of the probes (present set). The dotted line refers to $B_{n}^{P}$ (due to the plasma) and the full line, to the total $B_{n}$ $\left(B_{n} \equiv B_{n}^{P}-B_{n}^{R}\right)$.

If the sensors are placed on a circular surface, near the plasma (Figs. 6 and 7 ), the effect of the vacuum 
vessel is considerably smaller. This allows identification of the Mirnorv oscillation mode directly from the measured field.

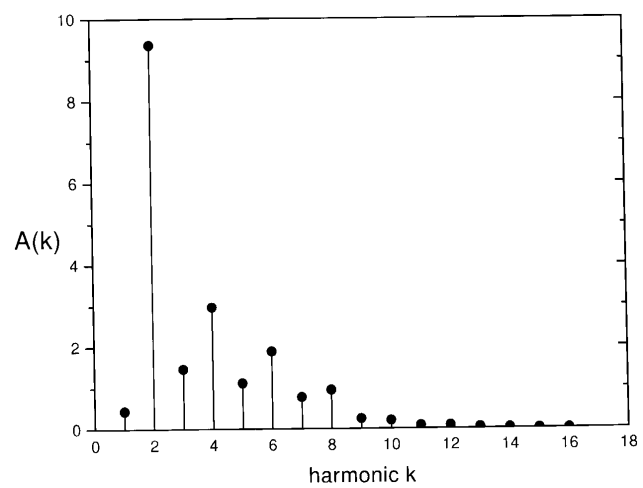

Figure 6. Spectrum of harmonics in reflected magnetic field at the position of the designed set of probes (near the plasma boundary).

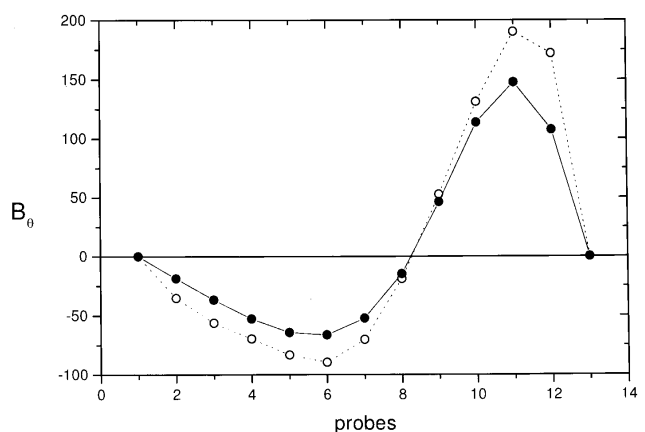

Figure 7. Poloidal field in the position of the designed set of probes. The dotted line refers to the field due to the plasma $B_{\theta}^{p}$ and the full line to the total $B_{\theta}\left(B_{\theta} \equiv B_{\theta}^{p}-B_{\theta}^{R}\right)$.

\section{Conclusions}

The magnetic field due to instabilities in a tokamak plasma is reflected in the conductive vessel wall. A method is developed here in order to find the reflected field. The method is based on expansion of vacuum field in series of toroidal harmonics.

This method is used to study the effect of the vacuum vessel on the Mirnov oscillation diagnosis in the tokamak TCABR.

The conclusion is that, if the sensors are placed near the vessel of rectangular cross section, the effect of the vessel is rather strong. A possible approach to interpretation of measurements is reconstruction of the vacuum magnetic field from external measurements. This reconstruction would use the same method of expansion of vacuum field in toroidal harmonics generated in the plasma. Only, in this case, the vacuum field is represented by toroidal harmonics generated in the plasma and the reflected field.

\section{References}

[1] Yu. K. Kuznetsov, I. C. Nascimento, R. M. O. Galvão and W. P. de Sá, Nucl. Fusion 38, 1385 (1998).

[2] R. V. Churchill 1963 Fourier Series and Boundary Value Problems chapter 3 McGraw-Hill Book Company, Inc. second edition. 